\title{
Options for intravenous iron supplementation in hemodialysis patients
}

\author{
Ji Yoon Kong ${ }^{(\mathbb{D}}$, Hyeon Seok Hwang ${ }^{(\mathbb{D})}$ \\ Division of Nephrology, Department of Internal Medicine, College of Medicine, Kyung Hee University, Seoul, Republic of Korea
}

Anemia is one of the most prevalent disorders in patients receiving hemodialysis (HD) treatment. The key pathophysiology of anemia in HD patients is decreased production of erythropoietin; regular hormonal replacement with erythropoiesis-stimulating agents (ESAs) plays central role in anemia treatment. The use of ESAs has yielded several beneficial effects, including improved quality of life, reduced need for blood transfusions and decreased anemia-related morbidity and mortality in chronic kidney disease (CKD). However, epoetin- $\alpha$ treatment with a target hemoglobin over $13.5 \mathrm{~g} / \mathrm{dL}$ was associated with increased risk of death and hospitalization for congestive heart failure and no additional benefit compared with a target hemoglobin of $11.3 \mathrm{~g} / \mathrm{dL}$ [1]. In the Trial to Reduce Cardiovascular Events with Aranesp Therapy (TREAT), darbepoetin- $\alpha$ treatment with a target $\mathrm{Hb}$ level of $13 \mathrm{~g} / \mathrm{dL}$ was associated with a significantly higher incidence of stroke in patients with diabetes and CKD [2]. Consequently, safety concerns about stroke and vascular thrombosis at higher doses of ESAs has emerged, and the use of ESA without use of iron supplement for anemia faced the limitation for optimal management of

Received July 29, 2020; Accepted August 24, 2020

Editor: Tae-Hyun Yoo, Yonsei University, Seoul, Republic of Korea Correspondence: Hyeon Seok Hwang

Department of Nephrology, Department of Internal Medicine, Kyung Hee University Medical Center, College of Medicine, Kyung Hee University, 23 Kyungheedae-ro, Dongdaemun-gu, Seoul 02447, Republic of Korea. E-mail: hwanghsne@gmail.com

Copyright (C 2020 by The Korean Society of Nephrology

(a) This is an open-access article distributed under the terms of the Creative Commons Attribution Non-Commercial License (http://creativecommons. org/licenses/by-nc-nd/4.0/), which permits unrestricted non-commercial use, distribution, and reproduction in any medium, provided the original work is properly cited. anemia.

ESA treatment can induce iron deficiency because of a massive transfer of stored iron in erythroid progenitor cells, which reduces the efficacy of ESA treatment. Most international treatment guidelines recommend monitoring iron status in patients receiving ESA treatment and iron supply is considered a supportive therapy to ESA administration in anemia management. However, in a recent trial, the proactive use of high-dose intravenous (IV) iron resulted in a significantly lower risk of death or major nonfatal cardiovascular events compared with that observed in a reactive, low-dose regimen [3]. This dosing strategy also resulted in a significantly lower dose of ESA and a lower incidence of blood transfusion, whereas the incidence of infection and hospitalization did not increase compared to a reactive regimen. These findings imply not only an active role of iron supplementation, but also suggest the compensational capacity against ESA treatment limitations. Therefore, it is necessary to identify an effective regimen for iron supplementation and to determine the efficacy and safety of current iron supplementation protocols.

The iron supplementation route can be oral or IV. Oral iron compounds are generally safe and physiologic with fewer costs. However, oral iron supplementation frequently causes gastrointestinal side effects, including constipation and bloating, which might reduce treatment adherence. In addition, owing to impaired intestinal absorption in patients receiving HD treatment, the effectiveness of oral iron treatment is decreased. While IV preparations are recognized as more effective, their use is associated with serious adverse events, including lifethreatening anaphylactic reactions in some individuals. Furthermore, IV iron preparations worsen oxidative 


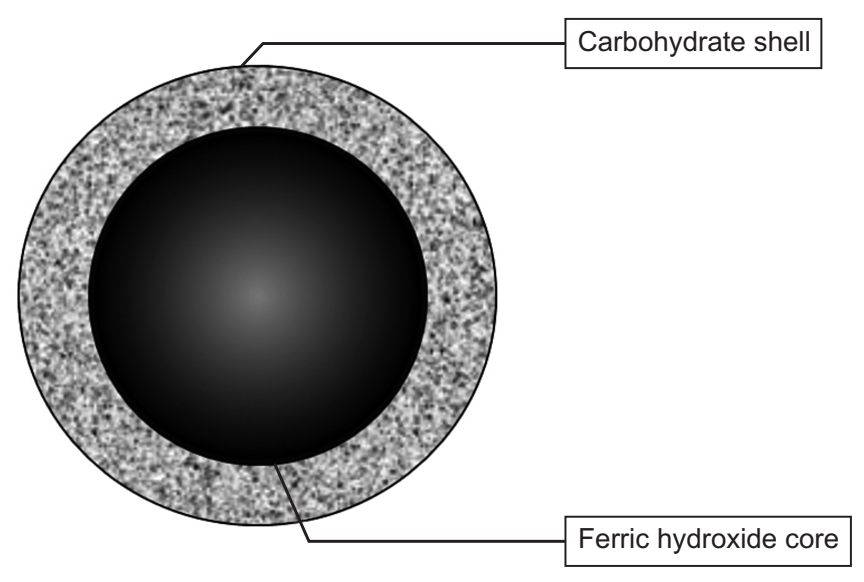

Figure 1. Structure of intravenous iron preparations. The ferric hydroxide core is surrounded by a carbohydrate shell, which stabilizes and determines the pharmacologic characteristics of iron supplements.

stress and systemic inflammation, which might increase the risk for infection, vascular calcification and cardiovascular disease. Therefore, oral and IV iron preparations both have advantages and disadvantages [4].

The current IV iron preparations include ferric gluconate, iron sucrose, ferric carboxymaltose, ferumoxytol and iron isomaltoside. They all share a similar core shell structure, because rapid release of large amounts of free iron in the bloodstream causes severe toxicity (Fig. 1). The ferric hydroxide core is surrounded by a carbohydrate shell, which prevents rapid release of iron content and promotes uptake into the reticuloendothelial system. Therefore, the carbohydrate shell determines the pharmacologic characteristics, efficacy of iron supplementation and drug bioactivity of the IV iron preparation.

Ferric carboxymaltose is an IV colloidal solution that contains ferric hydroxide core stabilized with carboxymaltose. It is designed to provide controlled delivery of iron into the reticuloendothelial system and to transport protein transferrin, inhibiting ionic iron release into the serum. The stability of ferric carboxymaltose enables the administration of high doses of iron and reduces the risk of adverse events. Therefore, we expect effective iron supplementation with minimum adverse consequences after IV ferric carboxymaltose treatment.

An extensive literature review identified several previous studies of IV ferric carboxymaltose treatment (Table 1) $[5-10]$. The Ferinject ${ }^{\circledR}$ assessment in patients with Iron deficiency anemia and Non-Dialysis-dependent Chronic
Kidney Disease (FIND-CKD) study was an open-label, multicenter, prospective, randomized study comparing IV ferric carboxymaltose and oral ferrous sulfate treatment in patients with nondialysis-dependent CKD who did not receive ESAs. This study found that IV ferric carboxymaltose corrected hemoglobin levels quickly and delayed the need for ESAs [5]. In an open-label trial randomized study of nondialysis-dependent CKD patients receiving ESA treatment, ferric carboxymaltose was more effective and better tolerated than oral ferrous sulfate for iron deficiency treatment [6]. Therefore, the superior efficacy of IV ferric carboxymaltose over oral iron therapy is supported by the integrated results of these two analyses. Ferric gluconate and iron sucrose have nearly identical characteristics and show valuable efficacy and safety for iron supplementation; they have been used widely for the past two decades in HD patients. REPAIR-IDA was a randomized, active-controlled study of nondialysisdependent CKD patients who were or were not receiving ESA treatment that compared ferric carboxymaltose and iron sucrose IV treatment. The study concluded that ferric carboxymaltose was not inferior in terms of treatment efficacy or safety and allowed more iron to be administered in fewer infusions [7]. In another study of nondialysis-dependent CKD or HD patients, IV ferric carboxymaltose was compared with standard medical care including IV, oral iron or ESA treatment. There was a significant increase in serum ferritin level and transferrin saturation, suggesting the superior performance of ferric carboxymaltose over standard medical care [8].

While several previous studies showed the positive results on ferric carboxymaltose, most studies enrolled nondialysis-dependent CKD or iron deficiency anemia. HD patients are most prone to iron deficiency through impaired iron absorption in the gastrointestinal tract and loss of blood during dialysis. Therefore, IV iron supplementation is important in HD patients and comparative studies of different IV iron formulations are needed. However, clinical studies on ferric carboxymaltose are sparse in HD patients despite its clinical usefulness. Lacquaniti and their colleagues published results on this topic in this issue of Kidney Research and Clinical Practice [10]. The authors demonstrated that ferric carboxymaltose treatment reduced the ESA dose and improved control of anemia with high levels of ferritin and transferrin saturation compared to ferric gluconate therapy. 


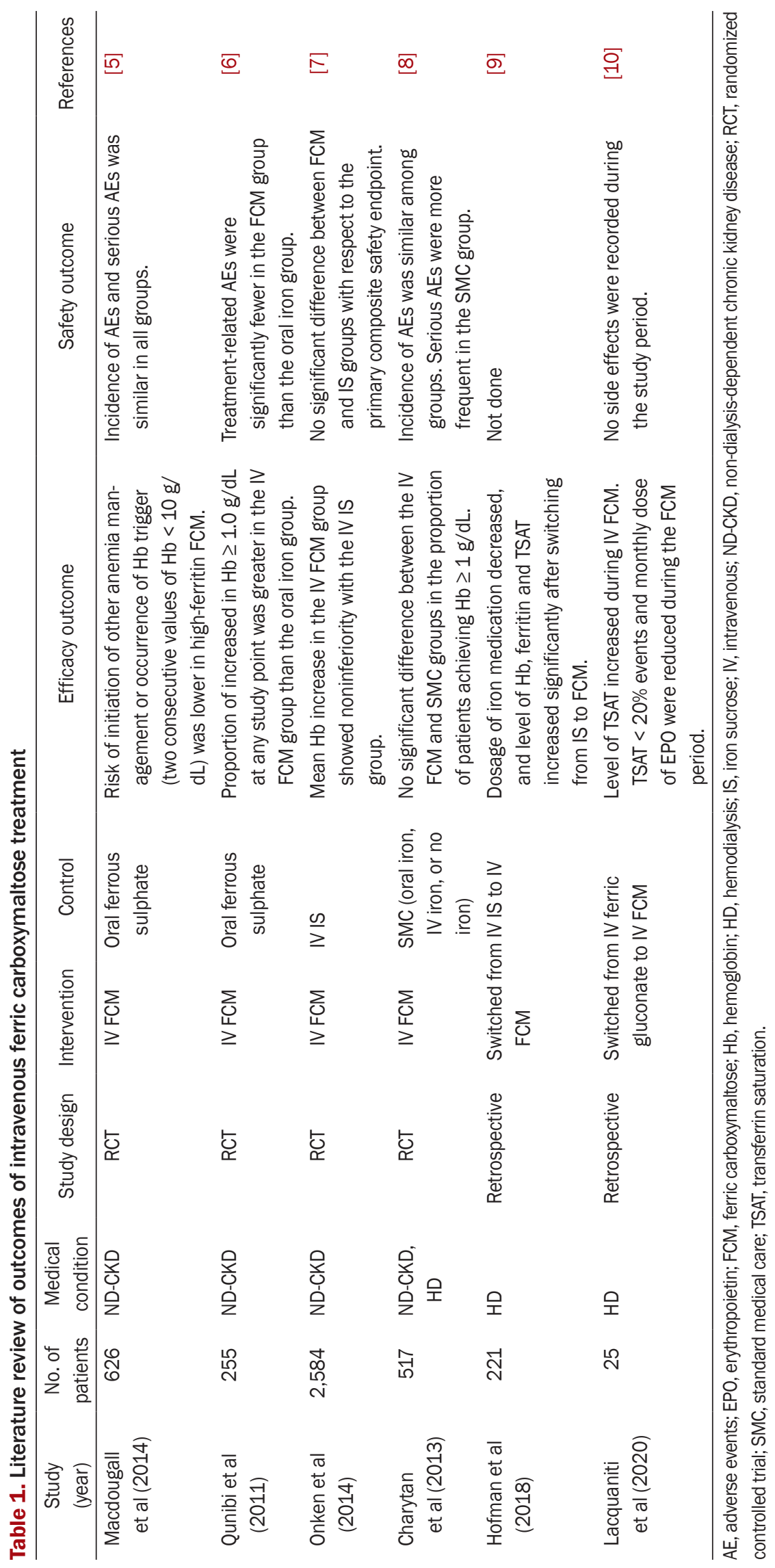


These findings are important, because the efficacy of ferric carboxymaltose has been validated in a susceptible population. In addition, the greater efficacy of ferric carboxymaltose was demonstrated compared with other IV preparations. Most previous comparative studies on ferric carboxymaltose focused on non-inferiority and did not directly compare effectiveness.

HD patients are exposed to oxidative stress, endothelial dysfunction and inflammation, which can be aggravated after IV iron supplementation. In this study by Lacquaniti et al [10], a decrease in erythropoietin resistance index was observed in the ferric carboxmaltose group, suggesting mediation of inflammatory processes. Based on this finding, we expect reduced inflammation-triggering effects and lower oxidative stress in ferric carboxymaltose treatment compared to ferric gluconate. However, biomarkers of inflammation such as C-reactive protein were not measured, and oxidative stress and endothelial dysfunction were not evaluated in this study. This limited our ability to assess adverse effects between ferric carboxymaltose and ferric gluconate.

Oxidative stress and inflammation are linked to increased risk of infection and cardiovascular disease in a clinical setting. Safety concerns about the adverse consequences of IV iron treatment require evaluation of major clinical end points. However, most clinical trials with IV iron preparations have focused on the efficacy of iron and hemoglobin correction and did not evaluate cardiovascular event or infection. To obtain better clinical outcomes, clinical studies on the safety profile of IV iron and ferric carboxymaltose are needed, including adverse clinical endpoints, in order to establish their superiority over other IV preparations.

IV iron treatment has become a popular anemia treatment due to recent prospective studies in patients with CKD and receiving HD treatment. As the role of iron therapy increases, several IV iron preparations have gained clinical importance. Ferric carboxymaltose shows better efficacy for treating anemia and iron deficiency in these populations and further studies are needed to compare the safety profiles of IV iron therapy.

\section{Conflicts of interest}

All authors have no conflicts of interest to declare.

\section{Authors' contributions}

Ji Yoon Kong participated in the data collection and wrote the manuscript. Hyeon Seok Hwang participated in the conception and provided intellectual content of critical importance to the work and technical support. All authors read and approved the final manuscript.

\section{References}

[1] Singh AK, Szczech L, Tang KL, et al.; CHOIR Investigators. Correction of anemia with epoetin alfa in chronic kidney disease. N Engl J Med 2006;355:2085-2098.

[2] Pfeffer MA, Burdmann EA, Chen CY, et al.; TREAT Investigators. A trial of darbepoetin alfa in type 2 diabetes and chronic kidney disease. N Engl J Med 2009;361:2019-2032.

[3] Macdougall IC, White C, Anker SD, et al.; PIVOTAL Investigators and Committees. Intravenous iron in patients undergoing maintenance hemodialysis. $N$ Engl J Med 2019;380:447-458.

[4] Vaziri ND, Kalantar-Zadeh K, Wish JB. New options for iron supplementation in maintenance hemodialysis patients. Am J Kidney Dis 2016;67:367-375.

[5] Macdougall IC, Bock AH, Carrera F, et al.; FIND-CKD Study Investigators. FIND-CKD: a randomized trial of intravenous ferric carboxymaltose versus oral iron in patients with chronic kidney disease and iron deficiency anaemia. Nephrol Dial Transplant 2014;29:2075-2084.

[6] Qunibi WY, Martinez C, Smith M, Benjamin J, Mangione A, Roger SD. A randomized controlled trial comparing intravenous ferric carboxymaltose with oral iron for treatment of iron deficiency anaemia of non-dialysis-dependent chronic kidney disease patients. Nephrol Dial Transplant 2011;26:1599-1607.

[7] Onken JE, Bregman DB, Harrington RA, et al. Ferric carboxymaltose in patients with iron-deficiency anemia and impaired renal function: the REPAIR-IDA trial. Nephrol Dial Transplant 2014;29:833-842.

[8] Charytan C, Bernardo MV, Koch TA, Butcher A, Morris D, Bregman DB. Intravenous ferric carboxymaltose versus standard medical care in the treatment of iron deficiency anemia in patients with chronic kidney disease: a randomized, active-controlled, multi-center study. Nephrol Dial Transplant 2013;28:953-964.

[9] Hofman JMG, Eisenga MF, Diepenbroek A, et al. Switching iron sucrose to ferric carboxymaltose associates to bet- 
ter control of iron status in hemodialysis patients. BMC Nephrol 2018;19:242.

[10] Lacquaniti A, Pasqualetti P, Tocco TCD, et al. Ferric car- boxymaltose versus ferric gluconate in hemodialysis patients: reduction of erythropoietin dose in 4 years of followup. Kidney Res Clin Pract 2020;39:334-343. 